Notfall Rettungsmed 2012 $15: 5-6$

DOI 10.1007/s10049-011-1555-4

Online publiziert: 18. Januar 2012

(c) Springer-Verlag 2012

\author{
U. Kreimeier ${ }^{1} \cdot$ H.-R. Arntz ${ }^{2}$ \\ ${ }^{1}$ Klinik für Anaesthesiologie, Klinikum der Universität München \\ ${ }^{2}$ Medizinische Klinik II, Kardiologie und Pulmonologie, Campus Benjamin Franklin, Charité, Berlin
}

\title{
Notfall + Rettungsmedizin 2012
}

Liebe Leserinnen und Leser,

mit dieser Ausgabe von Notfall + Rettungsmedizin dürfen wir die Organschaft für den Deutschen Berufsverband Rettungsdienst e.V. (DBRD) bekannt geben und begrüßen dessen Mitglieder im Kreis unserer festen Abonnenten. Eine Organschaft hat für die Mitglieder der Vereinigung nicht nur den Vorteil, regelmäßig über Fakten, Neuerungen und Trends im Bereich der gesamten Notfallmedizin informiert zu werden, sondern auch über eigene Mitteilungsseiten zu verfügen. Dies ist bei einer wissenschaftlichen Zeitschrift wichtig, da dort mit Ausnahme des Veranstaltungskalenders und der Anzeigen keine mitgliederbezogenen Informationen abgedruckt werden. Mit einer Organschaft wird über den Bezug der wissenschaftlichen Beiträge hinaus ein Informationsmedium angeboten, das eben diese Details für die Mitglieder kommuniziert.

\section{Bedeutung des Behandlungsteams}

Dr. U.K. Lindner, der erste Chefredakteur von Notfall + Rettungsmedizin, sah die Bedeutung des Behandlungsteams in der Notfallmedizin folgendermaßen: „Die Beherrschung des Notalls unter Umsetzung aktuellen medizinischen Wissens ist eine der zentralen Herausforderungen an das Notfallteam.... Das Zusammenspiel aller Kräfte fokussiert in der Qualität des modernen Rettungswesens, seiner Logistik und seiner Effizienz." (Editorial Heft 1/98). Unsere Zeitschrift verfolgt seit mehreren Jahren intensiv diesen ganzheitlichen Gedanken einer Zusammenarbeit aller Beteiligter zur Erzielung einer optimalen Effizienz und Ef- fektivität bei der Behandlung von Notfallpatienten, sowohl präklinisch als auch innerklinisch. Der Deutsche Berufsverband Rettungsdienst vertritt das Rettungsfachpersonal in allen den Rettungsdienst betreffenden Gremien und ist Mitglied der Ständigen Konferenz im Rettungsdienst (SKRD). Die „Ständige Konferenz" kümmert sich seit 1993 um die Weiterentwicklung des Rettungsdienstes in Deutschland und bezieht zu allen aktuellen rettungsdienstlichen Themen Stellung. Vorsitzender dieses Gremiums ist Dr. Dr. B. Dirks, Ulm.

\section{Ausblick 2012}

Mit der ersten Ausgabe im neuen Jahr dürfen wir Ihnen traditionsgemäß einen Ausblick auf das geben, was Sie, liebe Leserinnen und Leser von Notfall + Rettungsmedizin, konzeptionell und thematisch in diesem Jahr erwarten wird. Notfall + Rettungsmedizin wird in verbessertem Layout und in konstanter Qualität für über 6000 Abonnenten auch weiterhin wissenschaftliche Erkenntnisse auf neuestem Stand präsentieren. Wir werden das Konzept der Leitthemenbeiträge auf jeweils 3-4 Beiträge $\mathrm{zu}$ einem notfallmedizinisch hoch relevanten Themenbereich fokussieren. Neben vielen anderen wichtigen Beiträgen werden in Ausgabe 6/2012, die anlässlich des vom 18.-20.10.2012 in Wien stattfindenden internationalen Kongresses „Resuscitation 2012“ erscheint, „Kontroversen in der Reanimatologie" diskutiert werden. Sicherlich ein besonderes Highlight: Neue Erkenntnisse erfordern ständig das Überdenken von aktuellen Therapieempfehlungen, dem schließt sich unsere Zeitschrift mit einer entsprechenden Beitrags- reihe, verfasst von ausgewiesenen internationalen Experten auf diesem Gebiet, an.

\section{There is one more thing...}

Liebe Leserinnen und Leser von Notfall + Rettungsmedizin, alles, was Sie gedruckt in Händen halten, muss irgendwann einmal von jemandem niedergeschrieben, verfasst werden. Hierzu bedarf es motivierter Autorinnen und Autoren, die sich mit einer speziellen Thematik wissenschaftlich befassen, Untersuchungen planen, Ergebnisse zusammenstellen, kontextrelevante Literatur analysieren und als Beitrag für unsere Zeitschrift einreichen. Neu wird im kommenden Jahr eine Regelung sein, die Autoren wie Lesern entgegenkommt und auch ein neues Feature unserer Zeitschrift sein wird:

\section{Die 7er-Regel}

Sie besagt, dass ein eingereichtes Manuskript nicht mehr als 7 Schritte zu durchlaufen hat:

- Einreichung und Hochladen im Editorial Manager ${ }^{\circledast}$,

- Zuordnung der Gutachter und Erstellung der Gutachten

- Zusammenfassung der Gutachten und Mitteilung an die Autoren,

- Anfertigung der finalen Version,

- Annahme zur Veröffentlichung,

- Verfügbarkeit als Online-FirstBeitrag,

— Veröffentlichung in der Printausgabe.

Spätestens nach 7 Monaten soll der Beitrag - sofern zur Veröffentlichung angenommen - in der Printausgabe vorliegen. 
Wir sehen dies als einen weiteren wichtigen Schritt bei der Sicherstellung der Qualität, v. a. aber der Aktualität von Beiträgen an, und möchten potenzielle Autoren hiermit animieren, neben der in jüngster Zeit zunehmenden Zahl an Nur-Online-Journalen mit uns zusammen eine hochwertige, renommierte und topaktuelle deutsche nofallmedizinische Fachzeitschrift zu konzipieren, die international keinen Vergleich scheuen muss!

Ihre

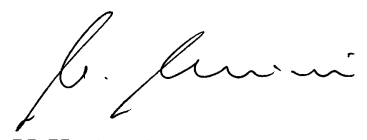

U. Kreimeier

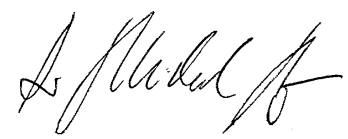

H.-R. Arntz

\section{Korrespondenzadresse}

\section{Prof. Dr. U. Kreimeier}

Klinik für Anaesthesiologie,

Klinikum der Universität München

Nussbaumstraße 20, 80336 München

uwe.kreimeier@med.uni-muenchen.de

\section{Thomas Nicolai \\ Pädiatrische Notfall- und Intensivmedizin}

Berlin, Heidelberg: Springer Verlag 2011, 4. Auflage, 540 S., (ISBN 978-3-642-20684-9)

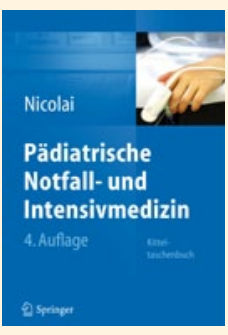

Die pädiatrische Intensivmedizin verlangt neben der klinischen Routine in einem sehr anspruchsvollen Patientengut ein profundes Wissen über diverse, teilweise ausgesprochen seltene angeborene und erworbene Pathophysiologien. In der Bewältigung dieser Aufgaben wird ein möglichst umfassendes, aber gleichzeitig auch präzise und kurz gefasstes Kitteltaschenbuch sicher ein wertvoller Begleiter für den in diesem Bereich tätigen Arzt/Ärztin sein.

Das vorliegende Kitteltaschenbuch hat eine klare Gliederung entsprechend der übergeordneten Organsysteme sowie eine zusätzliche Aufteilung nach intensivmedizinischen Techniken und Verfahren. Hier wird neben den intensivmedizinisch üblichen invasiven Verfahren auch die Beatmung von Kindern inklusive der Hochfrequenzbeatmung und Nicht-invasiven Beatmung, sowie der Bereich der Nierenersatzverfahren abgedeckt. Zusätzlich gibt es eigene Kapitel zu den Themen Vergiftungen und allergische Reaktionen, Thermische Verletzungen und Traumaversorgung. Darüberhinaus wird auf 128 Seiten eine aktualisierte alphabetische Übersicht der gängigen intensivmedizinisch relevanten Medikamente inklusive ihrer Dosierung und ggf. wichtiger Anmerkungen (z.B.: Dosisanpassung bei renaler oder hepatischer Insuffizienz) gegeben. Abgerundet wird das Buch von den aktuellen Empfehlungen zur Reanimation und dem Vorgehen in der post Reanimationsphase.

Im Detail zeichnet sich das Buch durch eine teils stichwortartige Abhandlung der Ätiologie, Definitionen, Klinik und Symptomatik aus; wichtige Bemerkungen zur Therapie, Diagnostik werden in farbig hinterlegten Tabellen aufgezeigt. Wo notwendig hat der Autor Anmerkungen zur (deutschen) Zulassungssituation von Pharmaka beigefügt, auch wenn diese manchesmal die Lücken und den programmierten off-label use in der Kinderintensivmedizin offenbaren.
Im letzten Kapitel werden dann noch Tabellen und Formeln der wichtigsten kardiozirkulatorischen und respiratorischen Parameter, zum Flüssigkeitshaushalt und Volumentherapie sowie Laborwerten geliefert. Ebenfalls stichwortartig werden sowohl die typischen Initialtherapien bei infektiologischen Erkrankungen mit und ohne Keimnachweis beschrieben.

Sehr hilfreich ist auch, dass am Ende des Buches eine ausklappbare Inkompatibilitätsliste der gängigen Arzneimittel angehängt ist, anhand derer man die Mischbarkeit von Medikamenten nachschlagen kann. Naturgemäss stellt die Anforderung an ein solches Buch immer einen Spagat zwischen Vollständigkeit und Reduktion auf das Wesentliche dar. Daher ist dieses Buch trotz der umfassenden Information auch eher als Nachschlagewerk und nicht als Lehrbuch zu verstehen. Denn der Leser braucht in aller Regel nicht eine erschöpfende Darstellung der meist kontroversen Diskussionen in der Fachwelt, sondern eine klinisch erprobte Vorgehensweise die auch in der Akutsituation angewendet werden kann.

Trotz der notwendigen komprimierten Darstellung bietet dieses Buch einen klinisch relevanten guten Überblick über die pädiatrische Intensivmedizin und ist somit dem (kinder)intensivmedizinisch tätigen Kollegen sehr zu empfehlen.

PD Dr. Thorsten Haas (Zürich) 